Huxley's instructive prologue and before the remainder of the text.

Around the centenary of Darwin's death in 1882 , there has been a plethora of texts concerned with the man, his theory, and its implications. But this must rank among the best in its breadth and scholarship and can be recommended unreservedly.

Alan E H Emery

\section{The Developmental Field Concept}

Edited by J M Opitz, J F Reynolds, and L M Spano. (Pp 476; £44.00.) New York: Alan R Liss. 1986.

This volume is an amalgamation of two issues of the American Journal of Medical Genetics (vol 21:1, 1985 and supplement 2,1986) and includes in excess of 50 articles, letters, and editorial comments which primarily address mechanisms of human malformations from a developmental field perspective.

The introductory chapter by the editor, Opitz, provides a valuable framework for the subsequent articles. In this chapter the developmental field concept is defined both from historical and current perspectives and the criteria for diagnosis of a malformation as a developmental field defect are clearly outlined. The articles that follow discuss a wide variety of malformations such as spina bifida, hypospadias and other midline defects, diaphragmatic defects, and DiGeorge anomaly, to name a few. These malformations, and others, serve to illustrate defects of specific developmental fields.

At first sight one might question the need to republish the papers in a book form as they are already in print and probably known to those interested in (dys)morphogenesis. However, on reading the volume, some merits of a collection of articles devoted to one concept become apparent. Collectively, the articles provide more compelling support for the 'developmental field hypothesis'. Furthermore, it is likely that, in this format, the developmental field concept can more readily be brought to the attention of readers outside the disciplines of clinical genetics and dysmorphology.

As might be expected in a volume of this kind, the quality of the articles is inconsistent. However, many of the contributors are well known and respected for their work in delineating mechanisms of birth defects. A collection of their articles, together with references, in a book form will be a useful addition to the personal libraries of those interested in the area of human fetal development.

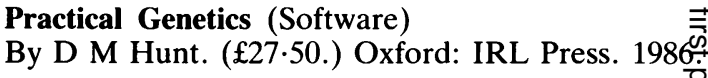

This is computer software designed as a tool fợ. teaching basic genetics. The programmes and th accompanying booklet describe the simulation of breeding experiments using the fruit fly Drosophit施 melanogaster and the mouse Mus musculus. Examples of recessive, dominant, and co-dominant inheritance, linkage, $\mathrm{X}$ linkage, homozygous lethality and sterility are used to show how the mutations are inherited. The programmes graphically display bot parents and all the progeny of each cross.

The package consists of six different programmes. an introduction, three programmes dealing wit $\overrightarrow{h_{1}}$ different types of inheritance in the fruit fly, \& programme concerned with monohybrid crosses i⿱艹 mice, and a chi square programme for statistical analysis.

The three Drosophila programmes are designe to set up crosses involving one pair of alleles (monohybrid), two pairs of alleles (dihybrid), an three pairs of alleles linked on the same chromosome allowing for the relative positions of the allelego along the chromosome to be mapped. In all three options, the programmes simulate the initial cross between a homozygous wild type fly and a homos zygous mutant fly. This is followed by a testcross and a reciprocal cross. Finally, the summary of the experiment is displayed and the user is asked to determine the mode of inheritance.

The mouse programme allows several othe inheritance patterns to be examined in sample crosses using only one pair of alleles. These include. co-dominance, sterility, and lethal genes. Several of: the mutations, and their inheritance patterns, arô similar to genetic disorders which occur in humansi

The final programme provides a chi square significance test on any of the data collected foo Drosophila or mouse. This is provided to examine whether the ratios or phenotypes observed among the progeny of a cross correspond to the expecte ratios, despite sampling error. In all cases, results of crosses can be printed out if a printer is available

Excellent graphical display of various mutations of mouse and Drosophila means that the pros grammes can be used to demonstrate different typeङ of inheritance and other concepts of basic geneticsTheir accuracy means that they can substitute for actual breeding experiments and yet convey the essential features of such experiments. Alternative ly, they can be used for individual or group study or to test the knowledge of the student. The programe mes can be run on either the BBC or IBM person computers. 